\section{Immuntherapie: Langzeiteffekt bestätigt}

\section{Die positiven Nachrichten zur Immuntherapie häufen sich. Das renommierte New England Journal of Medicine publizierte jüngst eine Studie über die Langzeiteffekte einer Immuntherapie bei Gräserpollenallergikern. Ergebnis: Der Behandlungserfolg bleibt auch noch Jahre nach Beendigung der Therapie erhalten.}

Die Immuntherapie zur Behandlung IgE-vermittelter allergischer Erkrankungen ist mittlerweile eine anerkannte Therapieform. Doch wie bei jeder neuen Therapieform gibt es auch bei der Immuntherapie noch offene Fragen, so z.B., ob der therapeutische Effekt auch nach Beendigung der Behandlung bestehen bleibt. Mehrere Untersuchungen erbrachten hierfür schon Belege. Die Ergebnisse einer neuen, sauber und aufwendig durchgeführten Studie der Arbeitsgruppe um St. R. Durham vom Royal Brompton Hospital und dem Imperial College at the National Heart and Lung Institute in London liefert nun neue, eindrucksvolle Daten, die den Langzeiteffekt der Immuntherapie dokumentieren.

\section{Studie läuft über 3 Jahre}

Die Arbeitsgruppe führte eine plazeboGräserpollenallergikern durch, wobei 32 der Patienten bereits 3 Jahre lang mit einem standardisierten Gräserpollen-Allergenpräparat (ALK-depot SQ) erfolgreich behandelt worden waren. Die Patienten wurden randomisiert in 3 Gruppen eingeteilt: Die Patienten der Gruppe 1 erhielten weiterhin die Verum-Immuntherapie $(n=16)$, die Patienten der Gruppe 2 wurden nach dreijähriger erfolgreicher Immuntherapie auf eine Plazebo-Immuntherapie umgestellt $(n=16)$ und 15 Patienten dienten als Kontrolle - sie wurden lediglich symptomatisch behandelt.

Über 3 Jahre sammelte man Daten zu den subjektiven und objektiven Krankheitsparametern dieser Patienten. Ergebnis: Der Behandlungserfolg kontrollierte Doppelblindstudie an 47 der Immuntherapie hält auch nach Abschluß der Behandlung noch über mindestens 3 Jahre an. So ließen sich z.B. bei den Scores der saisonalen Symptome wie auch bei dem Verbrauch an Akutmedikation der Patienten der Gruppe 1 und 2 keine statistisch signifikanten Unterschiede feststellen, während sich die Patienten der dritten Gruppe weiterhin mit ihren Beschwerden plagten.

Auch die objektiven Parameter wie die Spätreaktionen im Hauttest nach Allergenprovokation und die damit verbundene $\mathrm{CD} 3+\mathrm{T}-$ Zellinfiltration so- wie die Expression von Interleukin-4messenger-RNA zeigten nach $\mathrm{Ab}$ schluß der Immuntherapie keinen statistisch relevanten Anstieg in den Gruppen mit einer mindestens dreijährigen Immuntherapie - wenngleich sich hier eine gewisse Tendenz für eine Zunahme der Allergensensitivität bei den $\mathrm{Pa}$ tienten nach Abschluß der Immuntherapie ergab. Dies hatte aber keinen Einfluß auf das Wiederaufflammen der saisonalen Symptomatik.

Die Ergebnisse belegen, daß die Immuntherapie bei Patienten mit einer saisonalen Gräserpollenallergie auch noch mindestens 3 Jahre nach Beendigung der Behandlung anhält. Darüber hinaus unterstützt die Untersuchung auch die Erkenntnisse anderer Studien, nach denen die Immuntherapie den Langzeitverlauf der allergischen Erkrankung positiv beeinflußt. Die Autoren fordern daher, die Immuntherapie $\mathrm{zu}$ einem möglichst frühen Zeitpunkt einzusetzen und damit neben dem therapeutischen auch den präventiven Aspekt dieser Behandlungsform auszunutzen.

Durham StR, Walker SM, Varga E-M, et al. Long-term clinical efficacy of grass-pollen Immunotherapy. N Engl J Med 1999;

\section{Der Präventionsempfehlung einen Schritt näher}

Die Durham-Studie dokumentiert erneut die Wirksamkeit der Immuntherapie im therapeutischen Krankheitsmanagement bei Allergiepatienten und untermauert die Ausführungen des WHO-Positionspapiers zur spezifischen Immuntherapie, die letztes Jahr publiziert wurden. Darin erkennt die WHO erstmalig die spezifische Immuntherapie als „therapeutische Allergieimpfung“ bei den Indikationsgebieten allergische Rhinitis und allergisches Asthma an: „Die Immuntherapie ist die einzige Therapie, die den natürlichen Krankheitsverlauf allergischer Erkrankungen beeinflußt und die Entwicklung von Asthma bei Patienten mit allergischer Rhinitis verhindern kann.“ (Bousquet J, et al. J Allergy Clin Immunol 1998; 53, Suppl. 44).

Einschränkend wird jedoch festgestellt, daß die bisher publizierten Daten noch nicht ausreichen, um eine allgemeine Therapieempfehlung in Richtung Prävention, insbesondere hinsichtlich des „Etagenwechsels“ von der Rhinitis zum Asthma, durch die Immuntherapie aussprechen zu können. Hier erscheint die Durham-Studie als ein weiterer passender Puzzlestein, um ein neues Kapitel für die Einsatzmöglichkeiten der Immuntherapie aufschlagen zu können. Auch wenn die genauen Mechanismen noch nicht ganz verstanden werden, so belegen die in dieser Untersuchung gefundenen Daten jedoch eindeutig, daß ein lang anhaltender immunologischer und klinischer Effekt auch nach Beendigung der Therapie aufrecht erhalten bleibt. 